\title{
Chronologi|đąes Inhalitsperzeiфnis.
}

\begin{tabular}{|c|c|c|}
\hline Datum & Befes & ubturgung \\
\hline
\end{tabular}

\section{A. Beidoredt.}

1870, 4. Mai

1871, 7. Эunt

1871, 8. Juni

1871, 28. Ottober

1874, 17. Dat

1875, 6. Gebruat

1875, 14. MRã

1876, 11. Jaruar

1877, 27. Ianuar

1877, 27. Januar

1877, 30. Эanuar

1877, 30. Januar

1877, 10. Februar

1877, 10. హ̌ebruat

1878,18 . Эunt

1878, 24. Junt

1878, 30. Эunt

1878, 1. Suli

1879, 7. Эưt

1879, 21. Juli

1880, 5. Ypril (1886,

8. $\mathfrak{u} u(i)$

1885, 26. Mai

1889, 1. Mai

1890, 29. Juli

1891, 13. Mlai

1891, 1. Juni

1892 , 6. 9pril

1892, 20. Fptil
Berjonenftanbsgefeß far Bunbesange=! Ausißsto. borige im Guslanbe

Saftpfliotgeies

Ingaberpaplere mit \$rămien

Poftgejes mit sobellen

Stranbungsoronung

Perionenftandsgefes ac. (f. injem gtes gifter)

Bantgeies mit ben Robellen zc. (f. I fftem. Begifter)

Urheberredt an Puftern und $\mathbb{P R O}_{0}$ bellen zc. (f. inftem. Megifter)

Beridtoberfaffungsgefes zc. (f. fyitem. Regifter)

Cinfúbrungsgefę zum Ceriळtöber: faffung gefet

Bibllprojeforbnung ze. (f. fyftem. Me= giffer)

Cinfubrungsgefe zur glbilprozeforbs nung zc. (f. Inftem. Regifter)

Sontursorbnung 2c. (f. I gftem. Fegtfter)

Einfahrungegefes zur Kontursorbnung

Eertats toftengeies

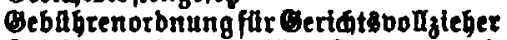

Bebahrenorbnung fitr Beugen unb Sadberitündige

Rectsantwaltsorbmung

Bebilbrenorbnung fit Regitsanmälte

Infectungsgejes

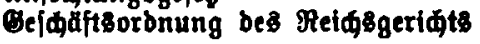

Peldstaffenfdeine

Benofleninaftsgefes

Bewerbegertatsogejeß

Batentgejes x. (f. inftem. Regifter)

Bebrauds multersejes

Telegraphengeies

Befellidaften mit bejŏrăntter ఏaftung
Saftplitgto.

Şnjaberp.

Boftes.

Strando.

$\$ 510$.

Banto.

Mufte.

(5)BO

EO. 6BO.

39D.

CE. 390.

20.

EC. RD.

083

BPOD.

800.

9uo.

MaOD.

Unfo.

भOSET氏D. 45

Mtafienfas.

Geno.

(S)S.

\$a10.

$\begin{array}{ll}\text { PalB. } & 38 \\ \text { Gebrinfte. } & 40\end{array}$

Tel@.

Bmbפo.
62

3

27

28

16

61

20

87

43

44

47

48

51

68

66

66

67

46

68

27.

11

54

29

10 


\begin{tabular}{|c|c|c|c|}
\hline Datum & Befet & abturyung & \\
\hline $\begin{array}{l}\text { 1894, 12. Mat } \\
\text { 1894, 16. Mat } \\
\text { 1895, 16. Junt } \\
\text { 1895, 15. Junt } \\
\text { 1896, 6. Jult } \\
\text { 1896, 18. Tugut }\end{array}$ & 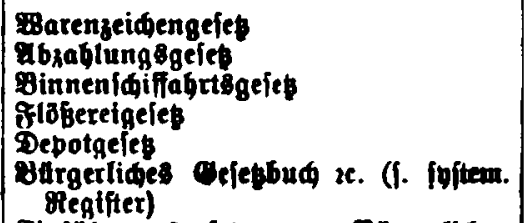 & 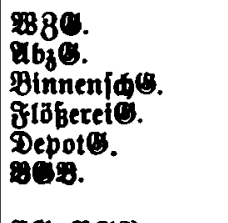 & $\begin{array}{r}41 \\
5 \\
17 \\
18 \\
23 \\
1\end{array}$ \\
\hline 1896, 18. Auguft & 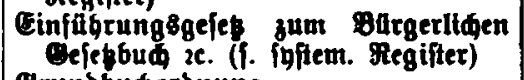 & EQ. POB. & 2 \\
\hline 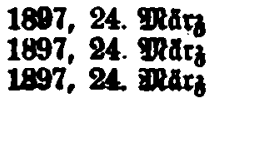 & 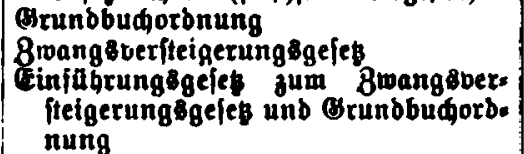 & $\begin{array}{l}\text { OBD. } \\
\text { 8BE. } \\
\text { ER. } 889 .\end{array}$ & $\begin{array}{l}58 \\
49 \\
50\end{array}$ \\
\hline $\begin{array}{l}\text { 1897, 10. Mal } \\
\text { 1897, 10. MRai }\end{array}$ & $\begin{array}{l}\text { Gandelsgefetbuth } \\
\text { Einfabrungsgefeh zum sanbelsgeieb= } \\
\text { bud }\end{array}$ & 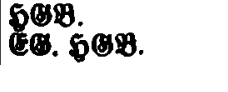 & $\begin{array}{l}8 \\
9\end{array}$ \\
\hline $\begin{array}{l}1898,17 . \\
1699,22 .\end{array}$ & 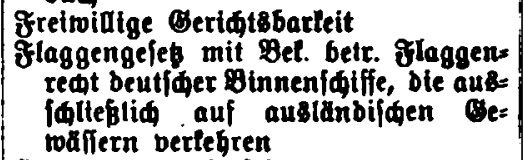 & $\begin{array}{l}\text { gres. } \\
\text { islaggents. }\end{array}$ & $\begin{array}{l}69 \\
19\end{array}$ \\
\hline 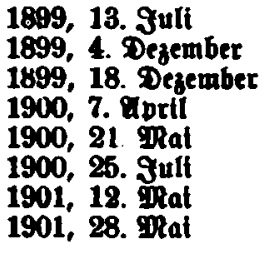 & 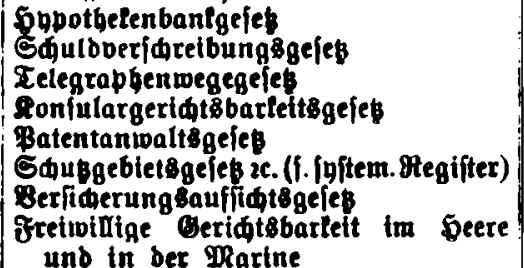 & 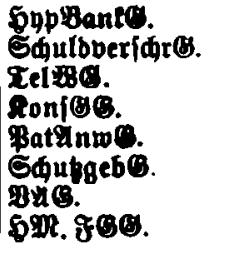 & $\begin{array}{l}21 \\
22 \\
80 \\
63 \\
89 \\
64 \\
81 \\
60\end{array}$ \\
\hline $1901,19$. & 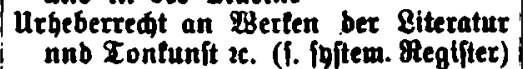 & gitues. & 33 \\
\hline $\begin{array}{l}\text { 1901, 19. Junt } \\
\text { 1902, 2. Эult } \\
\text { 1904, 6. Эult } \\
\text { 1905, 17. Эuft } \\
\text { 1905, 17. Jult }\end{array}$ & 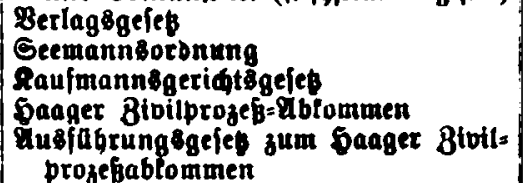 & 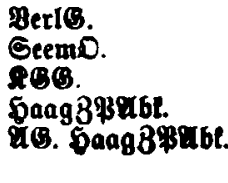 & $\begin{array}{l}35 \\
15 \\
55 \\
56 \\
57\end{array}$ \\
\hline 1907, & 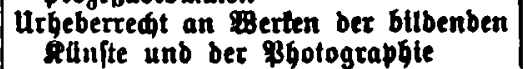 & Runftues. & 36 \\
\hline $\begin{array}{l}\text { 1908, 11. Mard } \\
\text { 1908, 27. Mat } \\
1908,30 \text {. Mal } \\
\text { 1908, 3. Sunt } \\
\text { 1908, 18. Mopember }\end{array}$ & 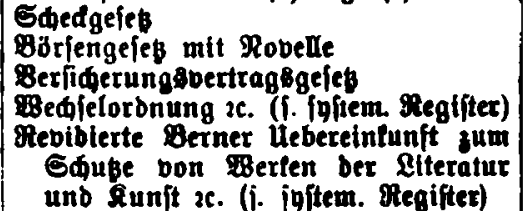 & 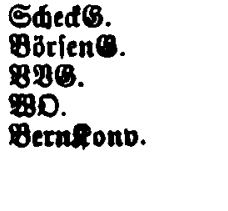 & $\begin{array}{l}11 \\
25 \\
32 \\
12 \\
84\end{array}$ \\
\hline 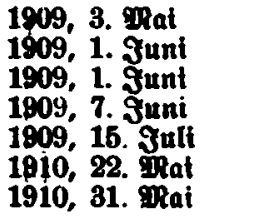 & 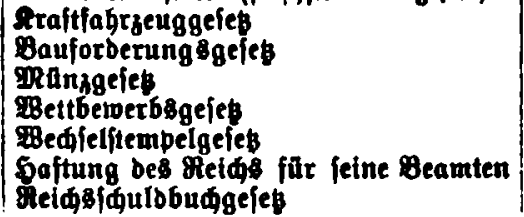 & 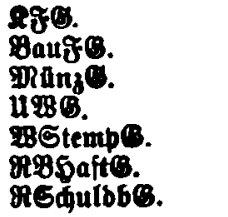 & $\begin{array}{r}4 \\
6 \\
26 \\
42 \\
13 \\
7 \\
24\end{array}$ \\
\hline
\end{tabular}




\begin{tabular}{|c|c|c|}
\hline Datum & Ceies & abthr;ung \\
\hline
\end{tabular}

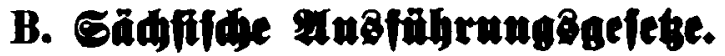

1879 , 1. Praz

1898, 28. SRai

1898, 31. Thai

1898, 18. Sunt

1899, 6. Эuli

1899, 26. Jult

1899, 8. Robember

1899, 10. Robember

1889, 11. Robember

1899, 22. Dejember

1900, 15. Juni

1900, 16. Juni

1900, 18. Juni

1900, 19. Эuni

1900, 26. Juni

1900,7 . Jult

1909 , 1. ఫुebruar

1909, 26. Februar

1909 , 6. Mat

Musflyongsgefes sum Geridts ber= fafiungsgefes

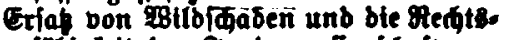
fabigleit ber Jagbgenofieniøaften

Hebibierte Befinbeorbnung

Yusfübrungegejet gum ofirgerltden Befébuate

Berorbnung zur Rusfabrung des Butgerftiben Celetbuches unb ber yo beffen Cine und Gubfabrung et: gangeuen Oelefe

Berorbmang zur Auffabrung ber Grunbbudorbnung

Mecorbnung ther bas Beglfterwefen bet ben \&utsgetimten

Berorbung zur Yusfá̧ning be San.

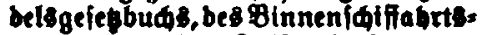
gefepes unb be gizberetgeieges

Berorbnung zur Rusfihtung bes 8126 bes Meidos geiezes iber bie fingelegen. beiten ber fretwolatgen Beridtabartett

chiet, bie Inlegung bon intinbelgelb betreffenb

Beles zur Qublabrung einiger mitt bem Burgerliden Bejegbude jujammen. bangenben Meidsgefete

Berorbnung zur Ausfiabrung ber Celebe uber ble Angelegengetten ber 8rets willigen Bertatsbarlett unb bes Sinterlegung swejens

Celes, ote 8mangsoouftredung in bas unbeweglide Bermögen betr.

Beroronning aur Gusfitbrung bee ges feplidien Beftimmungen ther bie Bwangovolftredung in bas unbes weglide Bermogen

Cefes jur RuBfibrung ber Bibliprozef: orbmang unb ber fonlur boronung

Gefes ther familtenanwartioaften

Qeie aber ble fintrorgeerjiegung

Forft: unb Felbftrafgeies

Beroronung zur Fubfibtung bes Ces febes aber ble filtiorgeergiebung

1910, 27. Mai

Defet uber bas \$fandeibgemetbe

\begin{tabular}{|c|c|}
\hline a0. C85. & 69 \\
\hline millbine. & 70 \\
\hline $\begin{array}{l}\text { MebCelo. } \\
\text { UE. BOS. }\end{array}$ & 7 \\
\hline צ'. BOB. & 73 \\
\hline å. 080. & 74 \\
\hline Reg8. & 70 \\
\hline MP. 508 . & 76 \\
\hline \&: 890. 8126 & 77 \\
\hline mos. & 78 \\
\hline ae. zoc. & 79 \\
\hline 48. 800. & 80 \\
\hline นo. 880. & 81 \\
\hline å. 889 & 88 \\
\hline AO. 8 BD & 88 \\
\hline 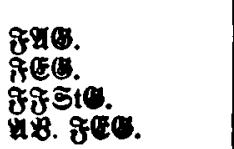 & $\begin{array}{l}84 \\
85 \\
8 \\
87\end{array}$ \\
\hline \$fanble & 188 \\
\hline
\end{tabular}

